\title{
Histoire et cultures de l'Asie centrale préislamique
}

\section{Frantz Grenet}

\section{OpenEdition}

\section{Journals}

Édition électronique

URL : https://journals.openedition.org/annuaire-cdf/15896

DOI : 10.4000/annuaire-cdf.15896

ISBN : 978-2-7226-0572-5

ISSN : 2109-9227

Éditeur

Collège de France

Édition imprimée

Date de publication : 30 décembre 2020

Pagination : 311-333

ISBN : 978-2-7226-0516-9

ISSN : 0069-5580

Référence électronique

Frantz Grenet, « Histoire et cultures de l'Asie centrale préislamique », L'annuaire du Collège de France [En ligne], 118 | 2020, mis en ligne le 01 avril 2021, consulté le 22 août 2022. URL : http://

journals.openedition.org/annuaire-cdf/15896 ; DOI : https://doi.org/10.4000/annuaire-cdf.15896 


\title{
HISTOIRE ET CULTURES DE L’ASIE CENTRALE PRÉISLAMIQUE
}

\author{
Frantz GRENET \\ Membre correspondant de l'Académie des inscriptions et belles-lettres, \\ professeur au Collège de France
}

Mots-clés : Asie centrale préislamique, colonies, Chine, Sogdiane, Inde, zoroastrisme, bouddhisme

La série de cours "Les colonies centrasiatiques en Chine (III ${ }^{\mathrm{e}} \mathrm{VIII}{ }^{\mathrm{e}} \mathrm{s}$.) : rôle social, religions, art » est disponible, en audio et en vidéo, sur le site internet du Collège de France (https://www.college-de-france.fr/site/frantz-grenet/course-2017-2018.htm), ainsi que la journée d'études «L'Ustrushana dans l'Antiquité et le haut Moyen Âge. Des marges de l'œkoumène au cœur du pouvoir abbasside » (https://www.collegede-france.fr/site/frantz-grenet/symposium-2017-2018.htm).

\section{ENSEIGNEMENT}

COURS - LeS COLONIES CENTRASIATIQUeS EN CHINE (III - -VIII $\mathrm{e}$ S.) : RÔLE SOCIAL, RELIGIONS, ART

Cours du 11 janvier 2018

Pourquoi avoir choisi ce sujet, après trois années d'étude des villes ? Plus que bien d'autres aires culturelles, l'Asie centrale s'est souvent projetée hors de ses limites, et son histoire n'est pas compréhensible sans une prise en compte de ce facteur. La projection de la «Haute Asie » a été militaire et migratoire, celle de l'Asie centrale «méridionale» a été commerciale, mais parfois aussi militaire (dans l'empire achéménide, dans l'empire abbasside). Pour ce qui la concerne, le facteur migratoire, notamment sogdien, n'a été reconnu que progressivement. Les études sur la « Route de la Soie » ont été longtemps dominées par le point de vue romano-byzantin (Ptolémée et l'information qu'il tirait de Maès Titianos, un marchand « macédonien » de Syrie ; puis, au VI ${ }^{\mathrm{e}}$ siècle, la guerre commerciale entre Byzance et la Perse, avec la tentative d'alliance turque). La somme d'Édouard Chavannes, Documents sur les Tou-kiue (Turcs) occidentaux (Saint-Pétersbourg, Académie Impériale des Sciences, 1903), bien que versant au dossier une masse de données chinoises, envisageait les rapports d'un point de vue encore très militaire et diplomatique ; les peuples d'Asie 
centrale sont considérés à demeure, dans leurs rapports avec les Chinois et les Turcs, mais très peu pour leur présence en Chine même.

L'existence de véritables colonies de peuplement sogdiennes en Chine a d'abord été reconnue à partir des sources chinoises, par Paul Pelliot, en 1916, pour la colonie du Lob-nor, puis par Edwin G. Pulleyblank, en 1952, pour celle de Mongolie intérieure; Pulleyblank a aussi mis en évidence le facteur politique des colons sogdiens dans l'histoire de la Chine des Tang (The Background of the Rebellion of An Lu-shan, Londres/New York, Oxford University Press, 1955). Peu après, l'importance des apports centrasiatiques dans la culture matérielle et littéraire de la période Tang était pleinement mise en lumière par Edward Schafer, dans The Golden Peaches of Samarkand. A Study of T'ang exotics (Berkeley/Los Angeles/Londres, University of California Press, 1963). À quoi s'ajoutaient les publications de collections de figurines d' "étrangers » trouvées en nombre considérable dans les tombes chinoises : d'abord Jane Mahler, The Westerners among the Figurines of the T'ang Dynasty of China (Rome, IsMEO, 1959), restée à ce jour la seule étude scientifique, mais avec des identifications approximatives sur des critères physiognomoniques dépassés. Depuis sont parus quantité de catalogues chinois avec de belles photos, mais sans véritables analyses. Les sources primaires proprement sogdiennes se réduisaient au dossier des Anciennes Lettres de Dunhuang (abrégé ci-après $A L$ ), éditées pour la première fois en 1931 par Hans Reichelt, commentées partiellement par Paul Pelliot puis par Walter Bruno Henning, mais posant de décourageants problèmes de lecture. On restait loin d'une perception globale de la présence centrasiatique en Chine.

La question a commencé à rebondir à partir des années 1990, avec trois incitations principales :

1) la redécouverte archéologique de la civilisation sogdienne en Sogdiane (notamment par les fouilles de Pendjikent);

2) un progrès sur les sources textuelles primaires: les Anciennes Lettres ont été mieux comprises et sont sorties de leur isolement grâce, notamment, au contrat sogdien de Turfan (639) publié par Yutaka Yoshida (voir ci-après) et aux inscriptions sogdiennes des passes du haut Indus attestant d'un commerce triangulaire SogdianeInde-Chine ; surtout, on a publié en masse les documents chinois des fouilles des cimetières d'Astana (registres de douanes, actes de la pratique), où des Sogdiens sont très fréquemment mentionnés ;

3) les tombeaux ornés des grands marchands sogdiens de Chine du Nord (dernier tiers du $\mathrm{VI}^{\mathrm{e}}$ siècle), la source principale mais non exclusive étant l'archéologie de sauvetage à Xi'an. En plus des nombreuses contributions parues en chinois, notamment des rapports de fouilles très bien illustrés, on a des études de Boris Marshak, Judith Lerner, Pénélope Riboud, et moi-même ${ }^{1}$.

La redécouverte archéologique de la civilisation sogdienne a été abordée en 20162017 pour les $\mathrm{V}^{\mathrm{e}}$ et $\mathrm{VI}^{\mathrm{e}}$ siècles, et le sera dans les années à venir pour son apogée aux $\mathrm{VII}^{\mathrm{e}}$ et $\mathrm{VIII}{ }^{\mathrm{e}}$ siècles. Dans le cours de cette année, nous examinerons en détails les sources de types 2 et 3 .

1. Dans la production chinoise récente en anglais, on signalera particulièrement, pour un essai de renouvellement de la mise en contexte historique, LI Yusheng, «Study of tombs of $H u$ people in late $6^{\text {th }}$ century Northern China », Newsleters di Archeologia CISA, vol. 7, 2016, p. 91-133. 
En conséquence de cette nouvelle situation documentaire, plusieurs ouvrages ont entrepris une approche globale du phénomène :

- Étienne DE LA VAISSIÈRE, Histoire des marchands sogdiens, Paris, Collège de France, 2003, 3e éd. 2016 (mais ne s'attardant pas beaucoup sur les tombeaux);

- le même É. DE LA VAISSIÈRE a coédité avec Éric TROMBERT le colloque Les Sogdiens en Chine, Paris, EFEO, 2004 ;

- Patrick Wertmann, Sogdians in China. Archaeological and Art Historical Analyses of Tombs and Texts from the $3^{\text {rd }}$ to the $10^{\text {th }}$ Century AD, Darmstadt, Philipp von Zabern, 2015 : assez en retrait sur le plan interprétatif, notamment pour les questions religieuses, mais solide et très complet à sa date (on s'y reportera pour les références bibliographiques non détaillées ici);

- et pour un renouveau de cette histoire vue de Chine: Valerie HANSEN, The Silk Road: A New History with Documents, New York, Oxford University Press, 2017, très au-dessus du niveau de la littérature surabondante qui paraît maintenant sur le sujet.

\section{La mise en place d'une immigration occidentale en Chine}

Les Sogdiens ont été les plus importants, mais ils n'ont pas été les premiers et ils ne sont jamais restés les seuls.

Les premiers témoignages, dans le Hanshu, font apparaître les marchands comme des parasites de la diplomatie. Rapport de 25 BC sur le Jipin (Kapisa et Gandhara; source : Hanshu 96 A p. 3886) :

Il n'y a pas de membre de la famille royale ou de nobles parmi ceux qui apportent des présents. Ceux-ci sont tous des marchands et des gens de basse condition. Ils veulent échanger leurs biens et commercer, sous le prétexte d'apporter des présents.

La Sogdiane est probablement citée dans le Hanshu parmi les royaumes soumis à la confédération nomade du Kangju, sous la forme Suxie (*sa-xat $=$ Sughd); elle l'est sûrement dans le Hou Hanshu. Il se pourrait donc qu'ici le commerce sogdien se glisse déjà derrière la diplomatie des suzerains nomades.

Malgré divers incidents, les échanges d'ambassades officielles avec le Kangju seront maintenus jusqu'à la fin du $\mathrm{III}^{\mathrm{e}}$ siècle, après quoi l'instabilité politique en Chine en fait fléchir le nombre pendant plus d'un siècle. Dès la chute des Han à la fin du $\mathrm{II}^{\mathrm{e}}$ siècle, la présence militaire et administrative chinoise dans le bassin du Tarim avait reflué et, par voie de conséquence, la transmission administrative des biens de prestige chinois (pas seulement la soie). Seule Loulan est conservée quelque temps. C'est sans doute alors que les Sogdiens s'imposent peu à peu plus à l'Est, créant un réseau continu d'implantations dans toutes les villes étapes entre l'Asie centrale et la Chine du Nord pour se rapprocher des marchés.

La première attestation sûre d'une colonie marchande établie à demeure est de 227 : peu après la chute des Han, des «seigneurs des Yuezhi et du Kangju » établis à Jincheng (aujourd'hui Lanzhou) au Gansu oriental accueillent l'armée de la nouvelle dynastie Cao Wei. Sous les noms de «Yuezhi » et de « Kangju » se cachent certainement des Bactriens et des Sogdiens, et les « rois» (wang) doivent être leurs chefs communautaires.

Outre cela, un indice indirect de la présence centrasiatique durant ces deux siècles précédant la grande crise chinoise du IV siècle est fourni par les premiers grands traducteurs d'écrits bouddhiques. An Shigao, arrivé à Luoyang en 148, sera dit plus tard issu d'une famille royale parthe $(A n=A n x i$ «Arsacide »); on a supposé qu'il 
était le descendant d'un prince otage indo-parthe, mais sans doute était-il en fait issu d'une famille marchande. L'origine princière ultime des introducteurs du bouddhisme en Chine est un stéréotype qu'on retrouvera plus tard à propos d'autres Centrasiatiques, peut-être favorisé par le fait que dans les langues iraniennes tout homme distingué est appelé bag (en sogdien vagh), selon les contextes « Dieu » ou «Sire»: l'équivoque iranienne sur les titres aura rejoint les manipulations mémorielles chinoises. D'une manière générale, tout étranger qui réussit en Chine est censé être issu d'une grande famille de son pays, ce qui donne d'autant plus de valeur à son ralliement à la culture chinoise. Un autre grand traducteur, Kang Senghui, était né au Tonkin au début du III $^{\mathrm{e}}$ siècle, dans une famille marchande sogdienne auparavant établie en Inde. Il est significatif de constater que ces premiers traducteurs n'étaient pas des Indiens, mais des Centrasiatiques rompus aux voyages et au multilinguisme (cf. aussi le Koutchéen Kumārajiva).

Cours du 18 janvier 2018

La première implantation sédentaire bien documentée par ses propres écrits est cependant d'une autre nature: les Indo-Bactriens du royaume de Kroraina, en chinois Shanshan (III $\mathrm{e}^{\mathrm{e}}-\mathrm{V}$ siècle), entre Niya et Loulan. Il est soumis au contrôle très théorique de la Chine depuis la garnison de Loulan, une colonie remontant aux Han, à l'extrémité est du royaume, et désormais le poste chinois le plus avancé. On connaît Kroraina à la fois par les chroniques chinoises et par les documents de pratique administrative retrouvés par Aurel Stein, majoritairement en prakrit local. Ces documents datent d'entre c. 240 et c. 360 . Leur langue indienne, le «prakrit de Niya », est en fait une langue artificielle à substrat local tokharien ou peut-être prototibétain, écrite en kharoshthi selon les pratiques indiennes de scribes qui portent des noms bouddhistes sanskrits. On pensait autrefois à une administration mise en place à la suite d'une véritable conquête kouchane (des expéditions sont effectivement mentionnées à plusieurs reprises, mais elles ne semblent pas avoir débouché sur des annexions) ; cependant, les noms des seigneurs sont locaux. Aujourd'hui, on suppose plutôt une immigration de paysans et de scribes depuis le Gandhāra, sans bouleversement des structures politiques locales, peut-être liée aux troubles lors du déclin de l'empire kouchan au III $^{\mathrm{e}}$ siècle. Ce sont des communautés plus agricoles que commerçantes ; Kroraina échange des ambassadeurs avec les royaumes voisins, mais les documents donnent peu d'indices quant à un commerce professionnel. Ce sont de petites gens qui font de petites affaires, en majorité du troc. La circulation monétaire suggère des échanges avec Khotan et avec l'empire kouchan alors en rétractation sur le Pendjab; pas avec Rome, contrairement à ce qu'on avait d'abord cru, et peu avec la Chine, sinon pour les besoins de la garnison de Loulan, d'où les visites occasionnelles de marchands « venus de Chine », pas forcément chinois, qui informent sur le prix de la soie. Comment l'expliquer ? On peut penser à un effet de distorsion des sources, avec une documentation plutôt au ras du sol, mais on a plus de mille documents. Cette situation pourrait en fait traduire un étiage du grand commerce caravanier dans une phase de double transition : après la fin simultanée (220-230) des empires kouchan et parthe dont la coexistence pacifique avait favorisé le transit transcontinental, puis la désintégration politique de la Chine du Nord après 300 ; et entre la prépondérance de la route du Sud (surtout indo-bactrienne) et l'émergence de la route du Nord (qui sera surtout sogdienne).

Cette population immigrée à Kroraina comprenait aussi des Bactriens, ce qu'indique l'onomastique, tandis que les documents chinois de la garnison de Loulan 


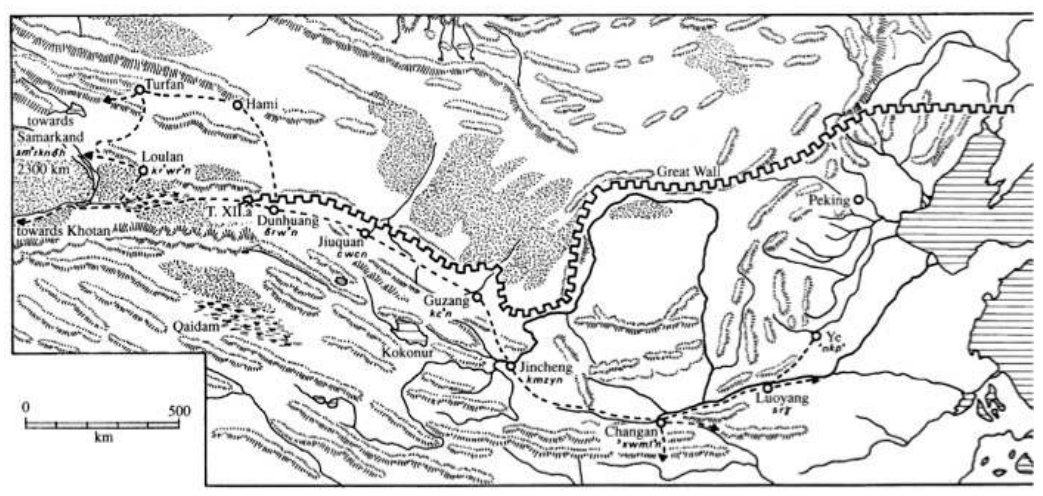

Figure 1 - La zone d'activité des marchands sogdiens d'après les Anciennes Lettres (les formes sogdiennes sont données en italiques).

montrent des Sogdiens comme fournisseurs occasionnels ${ }^{2}$. En miroir, les $A L$ montrent au début du $\mathrm{IV}^{\mathrm{e}}$ siècle les Sogdiens de Dunhuang en rapports commerciaux et matrimoniaux avec les Indo-Bactriens de Kroraina. Ceux-ci semblent avoir contribué à leur éducation commerciale, à en juger par les emprunts de vocabulaire indien en sogdien, particulièrement nombreux dans les $A L$. Aux $\mathrm{V}^{\mathrm{e}}$ et $\mathrm{VI}^{\mathrm{e}}$ siècles, les marchands sogdiens vont eux-mêmes prendre pied dans le nord-ouest de l'Inde, ce qu'attestent les inscriptions du haut Indus. On retrouve là des noms communs aux $A L$ (le nom clanique Kānak), ainsi que de nouveaux indices d'intermariages avec des Indo-Bactriens de Loulan.

\section{Les Anciennes Lettres}

Les marchands sogdiens dûment identifiés apparaissent sur le devant de la scène avec les $A L$, datables de $313-314^{3}$.

Les $A L$ sont un ensemble de huit lettres écrites sur papier, trouvées par Aurel Stein en 1907 dans une tour de guet à la sortie de la Grande Muraille, à 90 km à l'ouest de Dunhuang, $550 \mathrm{~km}$ à l'est de Loulan. Certaines étaient presque complètes, certaines fragmentaires, certaines encore scellées. Deux avaient été expédiées de Dunhuang, les autres de diverses stations au Gansu ; l'une avait pour destination Samarkand, les autres probablement Loulan (figure 1). Elles avaient été jetées

2. On a maintenant une lettre sogdienne trouvée à Niya, en gros contemporaine des $A L$, qui indique une présence sogdienne au-delà de Loulan : N. SimS-WILliAMS et Bi BO, « A Sogdian fragment from Niya », in Huaiyu CHEN et Xinjiang RONG (dir.), Great Journeys across the Pamir Mountains. A Festschrift in Honor of Zhang Guangda on his Eighty-fifth Birthday, Leyde/Boston, 2018, p. 83-104.

3. Je résume brièvement ici les deux cours consacrés à ce dossier, en renvoyant pour le reste à l'édition à paraître par Nicholas Sims-WiLliams, Early Sogdian Documents, Londres, Corpus inscriptionum Iranicarum, avec ma participation pour le commentaire. Pour une traduction non commentée des lettres I, II, III et V, voir pour le moment N. SIMS-WILLIAMS : https:// depts.washington.edu/silkroad/texts/sogdlet.html (consulté le 12 mars 2020). 
ensemble, l'hypothèse la plus probable étant qu'elles avaient été confisquées par la douane chinoise soucieuse d'éviter la dissémination des nouvelles sur la situation catastrophique en Chine.

Le contexte politique général est en effet marqué par les troubles en Chine du Nord, avec notamment, depuis 300, les rébellions des éléments Xiongnu installés dans les zones frontalières ou incorporés dans les armées. En 311, Luoyang, capitale des Jin occidentaux, est prise et pillée, l'empereur est capturé ; puis c'est au tour de Xi'an, à plusieurs reprises, et définitivement en 316. La Chine du Nord se retrouve politiquement fragmentée entre diverses dynasties généralement d'origine nomade (la Chine ne sera complètement réunifiée qu'en 589). Le poste avancé de Loulan est abandonné dès 330, tandis que le royaume de Kroraina disparaît à la fin $\mathrm{du} \mathrm{V}^{\mathrm{e}}$ siècle ; cette zone ne sera recolonisée qu' au $\mathrm{VII}^{\mathrm{e}}$ siècle, sous les Tang, par des émigrés de Samarkand et selon des modalités nouvelles, non pas une infiltration dans une population déjà présente, mais un peuplement par l'ensemble du corps social qui migre.

Ces textes sont d'une lecture difficile en raison des lacunes, de leur langue archaïque sans documents de comparaison, d'où de nombreux hapax; bien que sans doute dictés à des scribes ils sont d'une écriture négligée. Ceci est compensé en partie par le fait qu'il s'agit de textes visant l'efficacité (le scribe et le papier coûtaient cher), donc une fois qu'on a compris de quoi il s'agit, on peut deviner assez bien le détail.

$A L$ III est une lettre adressée par une femme à son mari qui est parti vers l'ouest en la laissant gérer ses affaires à Dunhuang et n'a plus donné de nouvelles depuis trois ans. Son associé est recherché pour dettes, ce qui affecte le sort de l'épouse et de sa fille, qui en sont réduites à se placer au service des Chinois. «J'aimerais mieux être la femme d'un chien ou d'un porc que la tienne !»

$A L \mathrm{I}$ : lettre de la même personne à sa mère demeurant sans doute à Loulan, fournissant quelques détails supplémentaires. Sollicitées de venir en aide aux déshéritées, la parentèle et les autorités sogdiennes de Dunhuang se sont dérobées et le seul recours est le prêtre (vaghnpat) qui fournira un chameau pour les aider à rejoindre Loulan.

Cours du 25 janvier 2018

$A L \mathrm{~V}$ : un rapport commercial à son patron par un marchand demeurant à Wuwei. Le patron est ici désigné comme sartpaw, «chef de caravane». Ce mot, plus tard transcrit sabao en chinois, désignera le chef communautaire, fonction que semble déjà annoncer celle de ce sartpaw qui a chargé son agent d'organiser l'émigration vers l'ouest de misérables compatriotes désignés comme "les Noirs », sans doute des paysans. L'auteur de la lettre trafique diverses denrées, dont le poivre long et la «blanche », sans doute le santal blanc. Il a été escroqué par d'autres marchands et aussi par les « Noirs ».

$A L$ VI (mal conservée) : un bref rapport également envoyé par un marchand à son patron. Il était chargé d'acheter à Loulan de la soie et du camphre, denrées qu'on s'attendrait à trouver plus facilement au Gansu : y aurait-il eu accumulation des stocks à Loulan et baisse des cours à cause de la raréfaction des caravanes vers l'ouest?

$A L$ II : la plus longue et la plus riche d'informations. "L'un des seuls documents à prouver l'existence d'un réseau sogdien, et non pas simplement d'un agrégat de petits marchands sogdiens, avec tout ce que la notion de réseau implique en 
termes de structure économique et sociale destinée à contrôler à distance les opérations commerciales ${ }^{4} \gg$.

Envoyée d'un lieu non spécifié, peut-être Zhangye, cette lettre est adressée simultanément à deux personnes, le père et le fils, proches parents et associés de l'envoyeur, restés à Samarkand. Elle a deux objets :

1) fournir un bilan d'activité. Ce n'est pas un bilan comptable : il ne porte pas sur du capital investi, mais sur les conditions de l'activité commerciale en général et sur les personnes employées pour y participer, que connaissent ses correspondants. Dans ce contexte est donné un compte rendu des récents troubles en Chine du Nord, dont l'envoyeur s'est informé en envoyant des agents. Les Sogdiens de la capitale sont morts de faim pendant le siège, la branche chinoise des affaires est complètement ruinée ; le circuit commercial se rétracte sur le Gansu, toujours dans le textile mais avec des produits bas de gamme ;

2) les dispositions testamentaires de l'envoyeur qui, âgé, n'envisage pas de rentrer au pays. Il a laissé son fils à ses correspondants, qui devront le marier et, pour lui éviter de partir sur les routes, gérer à son profit un capital de 60 ou 90 kilos d'argent (selon l'unité de poids employée), auquel s'ajouteront $800 \mathrm{~g}$ de musc d'une valeur équivalant à 30 kilos d'argent. Cette partie de la lettre fait connaître divers termes techniques d'interprétation parfois hypothétique quand ce sont des hapax: « capital », « intérêt », « dépôt non transférable», « document de transfert ». Ces familles qui gardent leur assise à Samarkand peuvent donc être très riches en capital, ce qui n'empêche pas leurs membres expatriés d'être confrontés à des réalités difficiles et parfois sordides.

\section{L'âge d'or des sabao}

La situation de crise continue aux IV et $V^{\mathrm{e}}$ siècles, à cause des troubles politiques aux deux bouts de la chaîne. Bien qu'alors peu documentées par les sources, les communautés sogdiennes survivent, quitte à se relocaliser ailleurs en Chine du Nord. La réunification brutale de la Chine du Nord par les Wei entraîne d'abord des dégâts collatéraux. En 439 à Wuwei les marchands sont faits prisonniers; le «roi de Soghd » qui obtient leur libération en 452 doit être un Kidarite ; est-ce début d'un plus grand intérêt des autorités de Samarkand pour leurs marchands ? À partir de cette époque on a à nouveau des échanges réguliers d'ambassades entre l'Asie centrale et la Chine du Nord, mais désormais la fiction politique du Kangju s'efface derrière les diverses principautés, au premier rang desquelles Samarkand. On est sans doute en train de passer au système qui dominera sous les Tang: des «ambassades» souvent fictives (pas toujours) qui sont en réalité des missions commerciales, le «tribut» étant l'habillage juridique d'une taxe. On a parfois des entrées assez surréalistes : des principautés minuscules offrent à la cour de Chine divers cadeaux précieux dont des danseurs, des femmes, des autruches qui alors existaient en Asie centrale, mais aussi des lions depuis longtemps disparus - et pourtant le terme chinois shizi les désigne sans ambiguïté ; étaient-ils acquis en Iran ou en Inde, acclimatés dans des parcs en Sogdiane?

4. É. DE LA VAISSIÈRE, Histoire des marchands sogdiens, op. cit., p. 43. 


\section{Cours du $1^{\text {er }}$ février 2018}

Un premier indice d'institutionnalisation de la présence sogdienne en Chine est fourni par le système des «neuf noms de famille », qui se stabilise au $\mathrm{VI}^{\mathrm{e}}$ siècle : Kang (Samarkand); An (Boukhara); Cao [Tsao] (Kabudhān) ; Cao occidental (Ishtikhān) ; Cao oriental (Ustrushana) ; Mi (Maymurgh) ; He (Kushāniya) ; Shi 1 $($ Kēsh = Shahr-i Sabz); Shi 2 (Chāch = Tachkent).

Samarkand a la primauté de prestige, même avant d'avoir acquis une réelle primauté politique à partir de c. 650 , et hérite de l'ancien nom du Kangju. Boukhara, le royaume le plus occidental, a récupéré l'ancien nom Anxi qui avait désigné l'empire parthe, ou Merv. Outre Kang et An, quatre noms se situent sur le cours même du Zarafshān que les Chinois appelaient la «Rivière des Caravaniers »: d'amont en aval, $M i$ (dont Pendjikent fut peut-être à un moment le chef-lieu), Cao, Cao occidental, He.

Seule la Sogdiane bénéficie d'un système classificatoire aussi détaillé ; les noms de famille attachés aux autres régions d'Asie centrale sont moins stables. Les Sogdiens sont désormais par excellence les $H u$, «Barbares d'Occident », même si cette dénomination ne leur est pas exclusive. Les Bactriens ( $L u o)$ font désormais pâle figure : peu sont mentionnés dans les documents ; ils semblent avoir une niche technique avec l'importation et la production de verre à $\mathrm{Xi}^{\prime} \mathrm{an}^{5}$, alors que les Sogdiens ne surent pas fabriquer le verre avant la conquête arabe. Les Chorasmiens sont associés aux Sogdiens mais sur la branche occidentale du commerce, vers la Volga (il y a cependant des ossuaires chorasmiens à Turfan). La concurrence persane semble limitée aux mers du Sud, avec un appui étatique sassanide, puis une colonie marchande à Canton.

On n'a que des données numériques parcellaires sur cette immigration. Les $A L$ mentionnent 100 chefs de famille dans une ville du Gansu dont le nom n'est pas conservé dans le document (c'est probablement Dunhuang). Plus tard à Xi'an, capitale des Tang qui compte alors un million d'habitants, ils seront très nombreux dans le quartier du Marché de l'Ouest; dès 641, 10000 familles de «Turcs orientaux » y auraient été installées, dont beaucoup devaient être des Sogdiens qui avaient des colonies en Mongolie méridionale. Étienne de la Vaissière a essayé de quantifier les données globales ${ }^{6}$ : au VIII ${ }^{\mathrm{e}}$ siècle, la Chine, d'après les recensements, compte 50 millions à 55 millions d'habitants (dont la moitié pour la Chine du Nord, 300000 à 600000 pour le Xinjiang et le Gansu); en regard, la vallée du Zarafshān, d'après les estimations, en compterait 1,3 millions à 2 millions, l'oasis de Bactres 300000 à 400000 , ce à quoi il faut ajouter les autres oasis occidentales. La proportion serait donc de un à dix entre l'Asie centrale occidentale et la Chine du Nord, ce qui rend beaucoup plus compréhensible le poids social des immigrants. C'est seulement à la période Yuan qu'on retrouvera une telle proportion de populations étrangères établies en Chine même.

Le bref âge d'or des sabao naîtra peu après. Dès 1962, Albert Dien avait attiré l'attention sur le statut très particulier de cette institution, au nom d'origine étrangère

5. Peut-être s'agit-il plus précisément de Juifs de Bactriane : S. KURINSKY, The Glassmakers: An Odyssey of the Jews, New York, Hippocrene Books, 1991, p. 255-256, 280-292.

6. «Early medieval Central Asian population estimates », JESHO, vol. 60, n 6, 2017, p. 788817, DOI : 10.1163/15685209-12341438. 
(il pensait à un nom indien), et, dans toute l'histoire de la Chine, la seule fonction de la hiérarchie mandarinale qui ait été réservée à des étrangers ${ }^{7}$. La question a été reprise par É. de la Vaissière et É. Trombert ${ }^{8}$. Depuis le III ${ }^{\mathrm{e}}$ siècle, on entend parler de chefs communautaires, par le texte sur Jincheng cité précédemment (les « rois »), puis par les $A L$ (les êpsār, « chefs »). C'est ensuite devenu une institution chinoise, mais véritablement opérante seulement sur un siècle. Elle semble apparaître sous les Wei dans la première moitié $d u V^{\mathrm{e}}$ siècle, et la dernière attestation effective est du milieu du $\mathrm{VII}^{\mathrm{e}}$ siècle; les mentions ultérieures dans les traités sur les institutions chinoises sont anachroniques. Elle a évolué. Dans les plus anciennes mentions, des documents administratifs de Turfan, vers 550, la forme chinoise est sabo (*sāt-b'a $\bar{a} k)$ < sanskrit sārthavāha, « conducteur de caravane », mais aussi « chef de la guilde des marchands ». À partir des Zhou du Nord (550-577), ils sont intégrés au système mandarinal, et c'est aussi de cette période que datent les premières tombes sogdiennes à décors spécifiques. Désormais connus sous la forme sabao (*sāt-paw', apparentée à la forme indienne mais refaite en bactrien et, de là, en sogdien: sārtpāw, «protecteur de caravane »), ils dépendent de l'Office de l'intendance des hôtes; il y a deux sabao à la capitale, un dans chaque région comportant plus de 200 foyers $\mathrm{Hu}$; ils sont l'avant-dernier des trente grades de la hiérarchie mandarinale ; dans les descriptions d'époque Tang, chaque sabao a un «bureau » comportant deux ministres du culte xian, un officier des gardes et un scribearchiviste, tous probablement sogdiens mais tous considérés comme mandarins, plus un personnel subalterne. Le culte xian est le nom sous lequel les Chinois ont désigné le zoroastrisme sogdien tel qu'il se présentait dans les communautés immigrées, le zoroastrisme pratiqué en Iran étant quant à lui désigné comme huo tian, «(religion) du Feu et du Ciel ». Les deux ministres du culte correspondent-ils au zōt et au raspīg du rituel zoroastrien? En tout cas presque toutes les figurations de scènes de culte, en Sogdiane comme en Chine, montrent deux prêtres officiant ensemble. Les sabao ont un signe vestimentaire caractéristique : le bonnet de tissu blanc en forme de fez, parfois bilobé, porté à la fois par les dignitaires figurés sur les tombeaux de pierre et par des marchands sur les peintures bouddhiques ${ }^{9}$.

Les généalogies laudatives dans les épitaphes des sabao ont joué sur l'équivoque du terme : quand on signale que le grand-père était sabao dans son pays natal, on peut penser que c'était simplement un sārtpāw au sens sogdien du terme, un marchand. Cependant, il est avéré que des ancêtres de sabao connus par les chroniques ou les épitaphes avaient déjà mené des carrières politiques sous les premiers Wei, avant l'établissement de l'institution: conseillers de l'empereur, administrateurs frontaliers, militaires. La rotation rapide des dynasties en Chine du Nord aura été un facteur d'intégration sociale pour les étrangers, l'apogée étant la prise du pouvoir par les Tang en 618 à partir de leur base de Taiyuan où les Sogdiens

7. «The sa-pao problem re-examined », JAOS, vol. 82, $\mathrm{n}^{\circ} 3$, 1962, p. 335-346, DOI : $10.2307 / 597645$.

8. «Des Chinois et des Hu. Migrations et intégration des Iraniens orientaux en milieu chinois durant le haut Moyen Âge », Annales. Histoire, Sciences Sociales, 59e année, nos 5-6, 2004, p. 931-969, DOI : 10.3917/anna.595.0931.

9. Rong Xinjiang, «Sabao or Sabo: Sogdian caravan leaders in the wall-paintings in Buddhist caves », in É. DE LA VAISSIÈrE et É. TromberT (dir), Les Sogdiens en Chine, op. cit., p. 207-230, pl. 18-19. 
étaient nombreux. On n'a pas d'attestation directe de leur rôle de diplomates chinois chez les Turcs dont l'empire s'impose en Haute Asie à partir de 552 (mais sur les tombeaux certaines scènes de rencontre entre Sogdiens et Turcs pourraient s'interpréter en ce sens). L'inverse est avéré : Maniakh en 568, probablement urtaq (fondé de pouvoirs commercial) du qaghan, était aussi son ambassadeur à Byzance. Le dynamisme diplomatique a changé de camp : en 484, c'est encore un marchand d'Apamée qui conseille les Hephtalites en faveur des Byzantins contre les Sassanides, mais, par la suite, les Syriens, ou du moins des usagers du syriaque, n'apparaîtront plus que dans un rôle religieux, parmi les Chrétiens et les Manichéens.

L'institution du sabao est progressivement dissoute sous les Tang (le dernier attesté par une épitaphe meurt en 646) et, finalement, seule subsiste la fonction subalterne de « chef du culte xian », les derniers témoignages de ce culte à Dunhuang étant de la fin du $\mathrm{X}^{\mathrm{e}}$ siècle. Cela ne veut pas dire qu'il n'y a plus de chefs communautaires, mais ils disparaissent de l'organigramme officiel au profit de la fiction des «cantons ralliés » soumis à la loi commune. "Cette nouvelle modalité d'existence sociale ne signifiait pas un déclin de l'influence sogdienne, bien au contraire, puisqu'elle faisait sortir une partie des Sogdiens du ghetto communautaire ${ }^{10} »$. Du reste, le milieu sogdien ou plutôt turco-sogdien va ressurgir en pleine lumière un siècle plus tard, lors de la révolte d'An Lushan.

\section{Les tombeaux sino-sogdiens}

\section{Cours du 8 février 2018}

Mais avant d'envisager cette période il nous faut examiner les grands tombeaux de sabao à iconographie caractéristique. Ce fut un phénomène brillant mais très temporaire ; ils appartiennent à une période encore plus courte que l'institution des sabao, le dernier tiers du $\mathrm{VI}^{\mathrm{e}}$ siècle, sous les Zhou du Nord et les Qi du Nord, puis les Sui ; il n'y en a déjà plus sous les Tang. Selon une hypothèse intéressante, des artistes auparavant au service des monastères bouddhiques et mis au chômage par la brève persécution de 574-581 se seraient reconvertis dans la commande funéraire des sabao, apportant avec eux leur talent et nombre d'éléments du langage symbolique bouddhique ${ }^{11}$. Ce sont les décors funéraires les plus riches de cette époque, venant juste après ceux de la famille impériale; il est probable que les sabao étaient parmi les éléments les plus fortunés de la population. Ces décors sont aussi, pour cette époque, les plus riches représentants d'un art figuratif sogdien encore très peu attesté en Sogdiane même.

Des Sogdiens (la majorité ?) ont continué à utiliser des ossuaires, trouvés notamment à Koutcha, Turfan, Urumqi. Certains sont identiques aux modèles sogdiens ou chorasmiens, d'autres sont adaptés à la forme des sarcophages chinois.

Du point de vue architectural, les tombeaux sont des tombeaux chinois creusés dans le lœss, avec une chambre funéraire et un dromos aménagé à partir d'une série de puits. La disposition du corps présente cependant des spécificités : dans les cas où l'observation est possible ils sont déposés sur la pierre du lit funéraire ou du

10. É. DE la VAissière et É. TROMBert, « Des Chinois et des Hu », op. cit., p. 961.

11. A. SHENG, «From stone to silk: Intercultural transformation of funerary furnishings among Eastern Asian peoples around 475-650 CE », in É. DE LA VAISSIÈrE et É. TROMBERT (dir), Les Sogdiens en Chine, op. cit., p. 141-180, pl. 17 (ici p. 167-168). 
sarcophage, sans cercueil de bois, ce qui indique une certaine conformité aux règles zoroastriennes qui proscrivent le bois, matériau conducteur de l'impureté. La même observation peut être faite à propos du mobilier funéraire, de type chinois, sauf qu'il ne comporte pas d'offrandes en nourriture ni en tissu. Il n'y a cependant pas d'indice net du rituel du décharnement (il y est fait allusion seulement sur un relief du tombeau Miho, voir ci-après, mais celui-ci est parvenu hors fouille). On connaît actuellement six tombeaux sogdiens entiers ou largement conservés, plus quatre tombeaux sans doute non sogdiens mais apparentés, et quelques bases dispersées ${ }^{12}$. Il est significatif que, même à Xi' an où on en a quatre datés ensemble à quelques années près, aucun décor n'a été exécuté par les mêmes artistes ${ }^{13}$.

Les plus anciens tombeaux d'étrangers datés par les épitaphes ont été trouvés dans les fouilles de Xi' an :

- Lidan, un «brahmane » (terme lâche qui peut désigner au sens large un Hindou), mort en 564 et qui avait auparavant vécu au Jibin (à cette époque, le Kapisa ou le Cachemire). Rien n'est spécifiquement hindou dans l'iconographie du sarcophage, du type chinois courant à cette époque, avec des éléments venus du bouddhisme ;

- Kangye, mort en 571. Son épitaphe chinoise le déclare « descendant d'un roi de Samarkand» et «grand maître céleste», titre par ailleurs inconnu, peut-être l'équivalent du «chef du culte xian» qui est un adjoint du sabao. Il termine sa carrière comme adjoint au gouverneur de Zhangye. Les reliefs du sarcophage sont un travail à la chinoise, en lignes incisées, extrêmement raffiné, sans thèmes zoroastriens reconnaissables malgré certaines hypothèses de chercheurs chinois (certains serviteurs ont des traits sogdiens, mais c'est l'entourage $\mathrm{Hu}$ normal pour un grand personnage à cette époque, et qu'on retrouve dans les tombeaux chinois). Le défunt et son épouse sont figurés comme un couple royal en procession, ou se délassant séparément, auquel cas le défunt fait face au spectateur, ce qu'on trouve déjà dans l'art funéraire chinois où le thème ouvre sur le monde de l'au-delà. Chinois également, mais d'origine bouddhique, sont le char à bœufs et le cheval sellé sans cavalier, ici vu de dos, qui semble être le cheval du défunt mené dans la procession funéraire. Une seule scène, où l'on présente au défunt des ballots de soie, fait référence à l'activité commerciale ou à son contrôle administratif.

Au même moment ou un peu plus tard apparaissent les premiers tombeaux à scènes typiquement sogdiennes. A Anyang (anciennement Ye) a été découvert en 1911 le premier ensemble apparu sur le marché. Les panneaux, dispersés entre Guimet, le musée d'Art Asiatique de Cologne et le Musée de Boston, ont été identifiés comme sogdiens en 1958. Stylistiquement, ils semblent venir tôt dans la série, peut-être dès avant 570 . On observe deux nouveautés :

1) la porte avancée qui remplace le dromos d'accès et qui, comme les parois des dromos chinois, comporte une procession - en l'occurrence, c'est clairement une procession funéraire zoroastrienne : deux prêtres au padām (couvre-bouche) sont figurés avec les ustensiles du čahärom, le rituel du quatrième matin après la mort (tisonnier, brûle-parfum, coupe à offrandes alimentaires, dont des pains, vase à vin.

12. Un autre tombeau (un lit funéraire en style incisé, sans épitaphe) est entré récemment au Musée national de Pékin. Il semble inclure des scènes de processions du Nouvel An. Il sera publié par Matteo Compareti et Li Sifei.

13. Hors images ou références spécifiques, on renvoie pour tous ces monuments au répertoire de Wertmann, mentionné en introduction du cours. 
La procession de gardes armés (cf. l'existence d'un « officier des gardes » parmi le personnel des sabao) amène des chevaux sellés et harnachés, ce qui révèle ici la fusion du thème chinois et du cheval sacrificiel sogdien attesté sur des ossuaires et sur la «Peinture des Ambassadeurs » de Samarkand ;

2) les panneaux du lit funéraire sont conservés sur trois côtés. Chacun montre au centre un cavalier s'avançant sous un parasol et, de part et d'autre, le même personnage festoyant. Le thème le plus reconnaissable est le Nouvel An zoroastrien, alors en juillet, d'où les treilles; il pourrait y avoir d'autres fêtes saisonnières. Pour Boris Marshak, influencé par la «Peinture des Ambassadeurs » de Samarkand, ce sont les «Rois du Monde ${ }^{14}$ », mais comme le personnage principal et ses commensaux masculins portent tous le bonnet du sabao, il s'agit plus probablement de la démultiplication d'un thème, attestée depuis par d'autres tombeaux : le sabao recevant ses compatriotes aux fêtes religieuses, ce en quoi il hérite d'une fonction royale iranienne. Les musiciens et les danseurs sont sogdiens, les musiciennes chinoises. Tous les hommes de marque sont habillés à la sogdienne, toutes les femmes à la chinoise, ce qui avait conduit Marshak à supposer qu'il s'agissait d'épouses chinoises, mais cette idée a été réfutée grâce à un texte débusqué depuis par Pénélope Riboud (un passage d'une histoire locale de Turfan inséré dans le Zhoushu): «en ce qui concerne l'habillement, les hommes suivent la mode des $\mathrm{Hu}$ et les femmes à peu près celles des Chinoises ${ }^{15}$ ». La raison pourrait être que les Chinois des villes associaient le costume sogdien aux danseuses d'agrément, profession socialement déconsidérée et dont devait se démarquer une Sogdienne de la bonne société.

Le lit funéraire d'An Jia (517-579) est, avec celui de Yu Hong (voir ci-dessous), l'un des premiers à avoir été trouvé dans une fouille régulière, à Xi' an en 2000. Comme d'usage, l'épitaphe chinoise n'affiche que la carrière administrative : les ancêtres étaient originaires de Boukhara, le père a vécu à Wuwei, la mère était chinoise ; An Jia est devenu sabao de Tongzhou, une ville à l'est de Xi' an, avant de terminer sa carrière comme grand commandant militaire.

Les reliefs du lit funéraire font un usage profus de la feuille d'or et des couleurs vives. On a parlé d'un art de baraque de foire, jugement que je ne partage pas (on oublie un peu trop ce qu'était la polychromie sur la sculpture classique et médiévale, et ce qu'elle est toujours sur la sculpture bouddhique vivante). Les seules scènes religieuses sont à l'entrée de la chambre funéraire : le rituel du čahārom est ici célébré par deux « prêtres-oiseaux », un motif qu'on va ensuite retrouver constamment.

\section{Cours du 15 février 2018}

Les prêtres-oiseaux (des hybrides d'hommes et de coqs portant le padām et tenant les baguettes rituelles) ne sont pas une adaptation des oiseaux célestes chinois, comme on l'avait d'abord cru, mais un motif proprement zoroastrien d'Asie centrale, attesté maintenant dès les environs de notre ère au Khorezm; ils représentent Parōdarsh, l'épiphanie animale du prêtre-assistant, un acolyte du dieu Srōsh sous la

14. B. MARSHAK, «La thématique sogdienne dans l'art de la Chine de la seconde moitié du $\mathrm{VI}^{\mathrm{e}}$ siècle », CRAI, 2001, p. 227-264 (ici p. 229-233).

15. F. GRENET et P. RIBOUD, «A reflection of the Hephtalite empire: The biographical narrative in the reliefs of the tomb of the sabao Wirkak (494-579) », Bulletin of the Asia Institute, vol. 17, 2007, p. 133-144 (ici p. 141). 

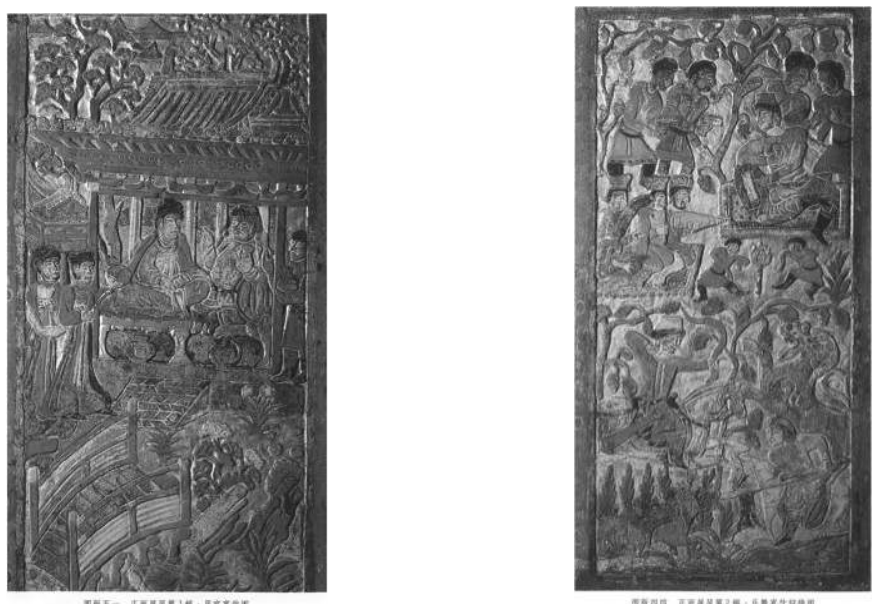

Figures 2a et $\mathbf{2 b}$ - Scènes du lit funéraire d'An Qia.

Source : Anjia tomb of Northern Zhou at Xi'an, Pékin, Cultural Relics Publishing House, 2003.

protection duquel l'âme est placée dans les trois jours après la mort, puis au matin du quatrième jour lorsqu'est célébré le čaharōm.

Tous les autres panneaux sont biographiques. Le but est de montrer le défunt, toujours identifié par son bonnet de sabao, soit dans le délassement aristocratique (figure 2a) soit dans des situations d'interaction diplomatiques. La scène du banquet à la sogdienne (figure $2 \mathrm{~b}$ ), sous la treille, montre des nains dansant, une chasse au lion (impossible dans les régions concernées, mais qui l'assimile à un roi sassanide, l'image du lion cabré étant copiée sur des plats). Les scènes d'interaction impliquent presque toujours des Turcs, car l'apogée de la carrière d'An Jia a coïncidé avec l'émergence des empires turcs après 550. Les entrevues se déroulent dans une atmosphère festive : chasse, toasts, musique mixte où le sabao joue lui-même, danse (les danseurs et musiciens sont toujours des hommes, à la sogdienne), jeu d'échecs ou de nard. An Jia aurait pu être chargé de missions diplomatiques par les Zhou (dans une scène, un personnage à couronne de type sassanide attend son tour : un roi sogdien ?), mais les négociations n'étaient pas forcément d'État à État: elles pourraient avoir pour but de sécuriser les routes caravanières. Il est clair que les exécutants des reliefs ont travaillé sur des instructions données par la famille et qu'ils ont voulu privilégier la partie itinérante de sa carrière.

Dans tout le répertoire des tombes sino-sogdiennes, seuls deux défunts n'apparaissent que pour leur carrière commerciale, et il se trouve que dans les deux cas, ils sont morts trop jeunes pour devenir sabao:

1) l'épitaphe sino-sogdienne (l'une des deux seules) d'un Nanayvande, tout récemment identifiée à Anyang ${ }^{16}$. On n'a pas le tombeau; mort jeune en 580, il

16. Bi Bo et N. Sims-Williams, "Another Sogdian-Chinese bilingual epitaph », BSOAS, vol. 80, 2017, p. 305-318. 
est désigné en chinois comme «marchand itinérant» (shangke), en sogdien, comme $x w \bar{a} \bar{c} \bar{a} k$, un hapax à l'étymologie incertaine mais qui pourrait être à l'origine du persan xwäjah, titre de respect à signification large ;

2) le lit funéraire d'An Bei à Luoyang. Fouillé illégalement, il a livré un décor incomplet et maintenant dispersé (la base est conservée au Musée municipal de Xi'an, les panneaux, dans une collection privée à Macao). L'épitaphe chinoise indique qu'il est mort en 589. Les reliefs, parmi les plus élégants de la série, ont sans doute été payés par son père qui était l'un des commandants de la garde impériale des Sui. Quand il est mort à 34 ans, An Bei n'occupait encore qu'un poste subalterne (et peut-être fictif ?) dans l'administration militaire. L'épitaphe loue ses qualités de marchand, ce qui est très inhabituel dans une épitaphe chinoise. Les quatre panneaux qui subsistent se répartissent en deux thèmes :

- le défunt à la tête d'une caravane. Tous portent une couronne de fantaisie, pseudosassanide - parce qu'on ne pouvait pas leur attribuer le bonnet de sabao ? - ; dans une deuxième scène, les personnages sont nimbés et on tient un parasol sur le défunt - est ce déjà le chemin du Paradis ?

- le banquet et la danse. L'un des banquets est clairement paradisiaque ; les buveurs sont des personnages bachiques directement inspirées du Kubera indien, qu'on rencontre aussi dans l'art sogdien.

Les thèmes religieux proprement zoroastriens prennent une place plus importante sur les trois prochains tombeaux que nous allons examiner, le quatrième ne relevant pas de cette religion.

\section{Le lit Miho}

Il est nommé ainsi d'après le musée japonais qui l'a acquis, car on n'a pas l'épitaphe. Il est apparu sur le marché de l'art américain à la fin des années 1980. D'après des rumeurs le tombeau aurait été découvert à Taiyuan au Shanxi, où effectivement a été trouvé plus tard en fouille le tombeau de Yu Hong (voir ci-après). Il peut paraitre étrange que rien n'ait été trouvé par des archéologues avant ces années, et on ne peut exclure que, pendant la Révolution culturelle et dans les temps qui suivirent, des tombeaux à iconographie manifestement étrangère aient été réenfouis ou exfiltrés par Hong Kong. Le point de vue idéologique s'est maintenant renversé : les Sogdiens sont très populaires chez les savants chinois et on les affiche dans les musées ; la Chine est aussi sûre d'elle-même qu'elle l'était sous les Tang, et la façon dont elle a su digérer l'apport sogdien lui semble préfigure la façon dont elle sait maintenant digérer l'économie de marché (on évite encore le mot «capitalisme »).

Le répertoire des panneaux recoupe largement ce qu'on a rencontré sur les exemplaires précédents : le banquet accompagné de danses, avec l'épouse habillée à la chinoise ; les scènes avec des protagonistes étrangers (mais ici sans interaction manifeste avec le défunt), où apparaissent des Turcs, mais aussi des souverains d'Inde du Nord. Il est difficile de décider si cette ouverture spatiale est biographique ou conventionnelle. Toutefois, un élément religieux proprement sogdien est introduit dans deux scènes de voyage : la déesse Nana avec des musiciennes et des danseuses (dans un temple ?) ; le « cheval sans cavalier » sous le parasol, thème déjà présent $\mathrm{y}$ compris dans les tombeaux purement chinois, mais ici adoré et surmontant des poissons ; ce pourrait être le cheval du dieu Tishtrya, lui-même associé à l'Oxus, peut-être en rappel de voyages où le défunt aurait franchi ce fleuve. 
Surtout, l'un des panneaux est consacré au rituel des obsèques, cas unique dans toute la série. En bas, l'épouse, la fille et les trois fils se recueillent dans un paysage montagneux ; c'est très probablement l'abandon du corps pour le décharnement, lequel ne supposait pas nécessairement l'usage d'une «Tour du Silence ». Comme on n'a pas conservé le corps, on ne peut pas vérifier si ce rituel avait été effectivement pratiqué. En haut, le prêtre célébrant le čahārom voisine avec le rituel de lamentations violentes ; l'épouse et la fille déposent le sedra, tissu votif, à l'entrée du Pont du Chinwad qui mène au Paradis, et ici la scène bascule horizontalement de l'espace rituel à l'espace mythique ${ }^{17}$.

Ce tombeau, dont on ne connaît pas la date, serait donc, avec le tombeau d'Anyang, le moins sinisé de l'ensemble.

\section{Le sarcophage de Wirkak à Xi'an, mort en $579^{18}$}

Cours du 8 mars 2018

Il y a deux épitaphes, sogdienne et chinoise, avec des textes très différents (l'épitaphe chinoise est comme d'ordinaire extrêmement stylisée). Voici l'épitaphe sogdienne ${ }^{19}$, complétée en italiques d'après les données de l'épitaphe chinoise :

C'était dans la période Daxiang des Grands Zhou, la $2^{\mathrm{e}}$ année, le $1^{\mathrm{er}}$ mois de l'année du Rat, le $23^{\mathrm{e}}$ jour [= 16 juin 579]. Or donc, il y avait un homme d'une famille de Kēsh habitant dans la ville de Kachān (Wuwei). Il obtint de l'empereur le titre de sabao de Kachān, et il était un notable dans le Sughdīkestān.

Il s'appelait Wirkak, fils de Wanūk, fils du sabao Rashtvande [la version chinoise précise pour celui-ci : "sabao dans son propre pays »].

Sa femme, née à Senpen (Xiping), s'appelait Wiyusi. Wirkak épousa sa femme à Senpen [en 519]. Il mourut ici à Khumdān (Xi'an) [âgé de 86 ans ; sa femme mourut un mois après lui].

Il n'y a pas d'être vivant qui naisse et ne doive mourir. Il est difficile de dépasser le terme de sa vie. Cependant, il est encore plus difficile dans le monde des hommes qu'un mari et sa femme se voient l'un l'autre durant toutes ces années, tous ces mois, tous ces jours, et qu'ils vivent ensemble tout ce temps aussi au Paradis.

Cette «maison des dieux» faite en pierre a été construite par Vrēsamanvande, Zhēmatvande et Frōtvande pour leurs père et mère, à l'endroit approprié.

La date indiquée au début est celle des funérailles du couple. «Un homme d'une famille de Kesh (Shi)»: on connaît par leurs tombes à épitaphes (sans décor sogdien) deux lignées Shi à Guyuan, entre le Gansu et Xi'an, qu'on suit sur six générations entre le $\mathrm{V}^{\mathrm{e}}$ siècle et 666. Les premiers ancêtres étaient « sabao dans

17. F. GRENET, «Le rituel funéraire zoroastrien du sedra dans l'iconographie sogdienne », in P. Gignoux, C. Jullien et F. Jullien (dir.), Trésors d'Orient. Mélanges offerts à Rika Gyselen, Paris, Association pour l'avancement des études iraniennes, coll. «Studia iranica », cahier $\mathrm{n}^{\circ}$ 42, 2009, p. 107-115.

18. Je renvoie aux articles que j'ai publiés à son sujet (voir les références en notes) et ne développe ici que les données que je n'y ai pas commentées.

19. Y. YoshIDA, « The Sogdian version of the new Xi' an inscription », in É. DE LA VAISSIÈre et É. TROMBERT (dir), Les Sogdiens en Chine, op. cit., p. 57-72. 
leur pays », ensuite on trouve un gouverneur de Xiping, des militaires, un interprète à la cour des Tang, un directeur des haras impériaux. Dans l'une de ces tombes, de petits objets ayant échappé au pillage suggèrent des contacts avec l'Occident (des imitations de monnaies sassanides, des monnaies byzantines et leurs imitations, un sceau sassanide). Bien qu'on n'arrive pas à raccrocher Wirkak à leur arbre généalogique, certains prénoms se retrouvent, et Yoshida suppose qu'il leur était apparenté. Le Sughdīkestān où Wirkak était un «notable» est une désignation non attestée par ailleurs: est-ce la communauté sogdienne de Wuwei, la Sogdiane, l'espace commercial sogdien au sens large ?

En plus des compositions habituelles incluant des prêtres-oiseaux, on a deux types de scènes : des scènes religieuses à contenu surtout eschatologique, et des scènes biographiques. Pour celles-ci il s'agit de six scènes occupant deux côtés. Plus nettement que sur les autres tombeaux, elles forment une séquence narrative, se lisant de droite à gauche ${ }^{20}$; elles montrent le défunt d'abord participant très jeune aux voyages caravaniers de son père ou de son grandpère, en rapport avec des souverains sédentaires et nomades de la mouvance Hephtalite/Rouran (figure 3), puis marié, établi comme sabao, festoyant avec ses collègues à l'occasion du Nouvel An.

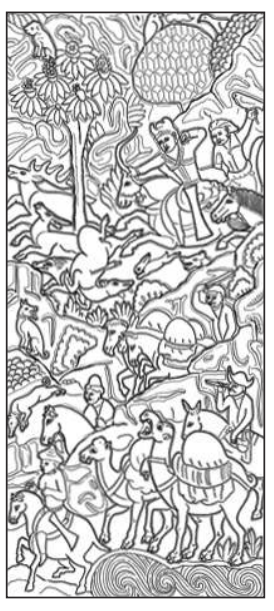

Figure 3 - Scène de caravane sur le sarcophage de Wirkak.

Noter la « longuevue », en fait un tube sans lentilles. Source : Shi Jun tomb of the Northern Zhou Dynasty, Pékin, Cultural Relics Press, 2014, p. 109. On est très loin du monde besogneux des $A L$. Aucun Turc n'apparaît, contrairement à ce qu'on voit sur le tombeau d'An Jia, strictement contemporain. Mais ce dernier, né en 517, a eu sa carrière active sous l'empire turc qui commence en 550, alors que depuis 535, Wirkak, né en 494, était déjà sédentarisé dans l'administration des sabao. Je supposerais volontiers que Wirkak et avant lui son père ou son grand-père avaient été les urtaq des souverains hephtalites ou alliés, avec lesquels ils apparaissent sur ces reliefs.

Les scènes religieuses sont au nombre de trois: une scène liminaire de prédication d'abord interprétée comme une prédication de Mani, mais qui a été rétablie comme figurant le message du Sauveur eschatologique du zoroastrisme ${ }^{21}$; deux scènes de conclusion, d'abord les angoisses des trois jours après la mort et le

20. F. GRENET et P. RIBOUD, «A reflection of the Hephtalite empire », op. cit.

21. Z. GULÁCSI, J. BEDUHN, « The religion of Wirkak and Wiyusi: The Zoroastrian iconographic program on a Sogdian sarcophagus from sixth-century Xi'an », Bulletin of the Asia Institute, vol. 26, 2012 [2016], p. 1-32. Ce message prêche l'abandon de la nourriture carnée, et par conséquent de la chasse. Quelques passages des livres pehlevis confirment l'existence d'une propagande végétarienne, certes minoritaire dans le zoroastrisme: Māh $\bar{l}$ Frawardīn rōz $\bar{l}$ Hordād, 40-41 (le végétarisme garantit de renaître jeune au Paradis), et surtout Dēnkard, VI.276 (il garantit de vivre vieux, ce qui précisément est advenu à Wirkak et à sa femme). 
secours par les anges «Bonne Pensée, Bonnes Paroles, Bonnes Actions », puis la montée au Paradis ${ }^{22}$.

\section{Cours du 15 mars 2018}

Deux tombeaux de style apparenté appartenaient à des représentants d'autres communautés auxquelles aurait pu s'appliquer la dénomination chinoise $\mathrm{ZaHu}$ «Hu mélangés ». Seul le premier est zoroastrien.

\section{Le sarcophage de Yu Hong (533-592) à Taiyuan}

L'épitaphe en chinois pose plusieurs problèmes. Le personnage n'est sans doute pas un Sogdien; il ne porte pas l'un des «neuf noms », mais est dit originaire du pays de « Yu » qu' on ne sait pas où situer, sinon vaguement à l'ouest. En fait, il fut d'abord un haut fonctionnaire des Rouran, comme avant lui son père et son grandpère. Le Weishu mentionne en 521 l'arrivée en Chine de six envoyés du roi des Rouran; le père ou le grand-père de Yu Hong aurait pu être l'un d'eux, et ce serait alors le seul cas de correspondance possible entre les personnages des tombeaux et ceux mentionnés par les chroniques. Les Rouran (autrement transcrits Juan-juan) étaient une confédération nomade alliée aux Hephtalites, défaite par les Turcs peu avant ces derniers, et qu'on voit plus tard réapparaître sous le nom d'Avars dans la région danubienne.

Yu Hong a accompli pour les Rouran une mission diplomatique chez les Tuyuhun (au Tibet oriental) et en Perse (dès l'âge de 13 ans nous dit-on, en 546 ! Comprenons qu'il accompagnait son père). En 550, il passe au service de la dynastie chinoise des Qi septentrionaux, sans doute, mais ce n'est pas dit, parce qu'alors l'empire Rouran a été soumis par les Turcs. Au total, il aura servi trois dynasties et dix empereurs. En 580, il reçoit un poste dans l'administration des sabao, mais en fait il semble avoir surtout occupé des fonctions de gouverneur militaire, ce qui laisse supposer que ses attaches étaient plutôt tribales que commerçantes.

Le décor a, semble-t-il, été exécuté en deux phases : d'abord des peintures à la mort de Yu Hong en 592, puis des reliefs peints quand sa femme l'a rejoint au tombeau en 598.

Les peintures, de style "sassanidisant", comportent des scènes de délassement aristocratique, de chasse, mais aussi une figure bachique évoquant le Paradis (déjà rencontrée sur le tombeau d'An Bei). L'un des panneaux sculptés montre le foulage du raisin, avec d'évidents modèles romains (cf. le sarcophage de Constance à Rome). Les autres reliefs relèvent d'un répertoire plus habituel, notamment la chasse, avec trois protagonistes bien différenciés ethniquement : le Turc au chameau, l'Arabe ou le Perse au dromadaire, l'Indien du Nord (ou le défunt lui-même ?) à l'éléphant ; les animaux chassés sont toujours des lions figurés comme sur les chasses royales sassanides. Certaines particularités - la longue barbe du défunt, les regalia dont il est affublé - renvoient aussi aux conventions sassanides. Moins encore qu'ailleurs, la frontière entre les plaisirs d'ici-bas et les plaisirs paradisiaques n'est nette ; l'emploi

22. F. GRENET, P. RiBOUd et YANG Junkai, «Zoroastrian scenes on a newly discovered Sogdian tomb in Xi'an, Northern China », Studia Iranica, vol. 33, 2004, p. 273-284; F. GRENET, « More Zoroastrian scenes on the Wirkak (Shi Jun) sarcophagus », Bulletin of the Asia Institute, vol. 27, 2013, p. 1-12. 
du halo ne semble pas consistant. Tout de même, un ensemble de trois scènes combinées pour créer un effet «théâtral » de profondeur semble bien évoquer le salut individuel : à droite, on amène le cheval sellé, et peut-être aussi le tissu de la sedra; à gauche, un jeune cavalier à riche couronne, tenant une grenade, pourrait être Mithra, juge des morts, recevant le cheval sacrificiel qui lui est particulièrement destiné ; au milieu, à l'arrière, une scène de banquet où sont concentrés les signes d'un banquet paradisiaque. C'est la seule scène où l'on voit des femmes, dont l'épouse du défunt (ce qui là aussi rappelle l'art sassanide où les femmes n'apparaissent pas dans des fonctions sociales). Les époux sont couronnés, ainsi que les femmes à droite (les Fravashis ?). Tous sauf les époux ont le halo.

Au total, ce décor est à la fois très raffiné et assez atypique ; il semble que $\mathrm{Yu}$ Hong ait été marqué par le séjour effectué dans sa jeunesse dans l'Iran de Khosrow Ir.

\section{Le « Lit Kooros»}

Le dernier exemple que nous examinons est le «Lit Kooros », trouvé dans une fouille clandestine à Tianshui à l'extrémité est du Gansu: l'épitaphe n'est pas conservée. Il a été brièvement exposé au musée Guimet en 2004, publié, puis il a disparu de la circulation.

Certains thèmes sont habituels : la chasse dans la steppe, le char à bœufs (avec la présence de nains), le banquet à la chinoise. D'autres sujets sont complètement originaux : le banquet à la sogdienne est carrément bachique, le défunt étant figuré ivre, ce qui serait inconcevable dans un cadre social chinois. Deux sujets religieux sont sans parallèles dans ce répertoire : Kubera sur son éléphant (une iconographie attestée en Inde, bien que rarement, avec là aussi une scène d'ivresse très expressive) ; Sūrya-Āditya à quatre bras, considéré comme un avatar de Vishnu (les eaux peuplées de makaras rappellent la figuration symbolique de l'Enfer zoroastrien sur le sarcophage de Wirkak, mais ici le thème renvoie plutôt à la purification de l'Océan primordial par Vishnu avant la création du monde).

La filiation avec l'iconographie dionysiaque du Gandhara a été particulièrement étudiée par Catherine Delacour ${ }^{23}$, qui pense que le défunt descendait d'une communauté indo-grecque. J'y verrais plutôt un Vishnuite, dont en l'absence d'épitaphe l'affiliation ethnique nous échappe (il y a des noms vishnuites aussi chez les Sogdiens). Une preuve par la négative est l'absence des brûle-parfum à la sogdienne et des prêtres-oiseaux (bien qu'il y ait des créatures fantastiques).

23. «Une version indienne du triomphe de Dionysos ? Essai d'interprétation de quelques-uns des panneaux historiés d'un monument funéraire chinois en pierre du VI $\mathrm{V}^{\mathrm{e}}$ siècle de notre ère », Monuments et mémoires de la Fondation Eugène Piot, vol. 84, 2005, p. 65-98. Pour une présentation plus neutre, voir C. DELACOUR et P. RIBOUD, Lit de pierre, sommeil barbare. Présentation, après restauration et remontage, d'une banquette funéraire ayant appartenu à un aristocrate d'Asie centrale venu s'établir en Chine au VI siècle (Musée Guimet, 13 avril24 mai 2004), Paris, Musée Guimet, 2004. Le «Lit Kooros » est très peu commenté dans l'ouvrage de WerTMANN, op. cit. 


\section{Les activités des Sogdiens de Chine}

\section{Cours du 5 avril 2018}

La diversité professionnelle des Sogdiens installés à Turfan et Dunhuang a été étudiée par Étienne de la Vaissière et Éric Trombert ${ }^{24}$, d'après des recensements locaux opérés par les autorités chinoises : des métiers liés au commerce (aubergistes, agrafeurs de pieds de chameau), mais aussi des tanneurs, des maroquiniers, et surtout une majorité d'agriculteurs, parmi lesquels des viticulteurs.

Les figurines de terre cuite à images d'étrangers, globalement datées de la période Tang, ne se prêtent certes pas à une statistique des métiers, la sélection tenant beaucoup compte du pittoresque. Les principales catégories du répertoire sont :

- le chameau bâté à la selle de bois, chargé de divers ustensile et d'outres, mais pas de rouleaux de soie ; souvent les traits caractériels du marchand intéressent plus que la marchandise elle-même. En plus d'importer du vin en Chine, les Sogdiens y ont introduit la viticulture ${ }^{25}$;

- le colporteur individuel ;

- une composante massive est représentée par les maquignons sogdiens ou turcosogdiens, qui, à partir du VII ${ }^{\mathrm{e}}$ siècle, se sont arrogé le monopole de la vente des chevaux et des chameaux aux haras impériaux ;

- les animaux de chasse importés en Chine : lévrier, faucon, guépard, caracal (?) ;

- les acrobates-danseurs (les seuls pour lesquels on reconnait d'autres types ethniques: Indiens du Sud, Asie du Sud-Est, peut-être Syriens); les nains, qui faisaient aussi l'objet d'un commerce ;

- les musiciens et musiciennes, qui se produisaient notamment dans les bars du quartier est de Xi'an. Une remarquable peinture trouvée dans une tombe de Xi'an datant de 740 montre, en vis-à-vis, un orchestre de femmes chinoises (figure 4a) et un orchestre d'hommes centrasiatiques (figure 4b), parmi lesquels des Sogdiens (dont le personnage à la Balzac qui chante sans instrument $)^{26}$.

À ce propos il faut signaler l'importance du commerce des femmes esclaves. À Turfan un recensement de la fin du VIII ${ }^{\mathrm{e}}$ siècle indique très probablement l'existence d'une sorte d' «école de geishas »: deux maisonnées où les noms sont sogdiens ou traduits du sogdien, avec un musicien, neuf serviteurs libres, 68 esclaves des deux sexes dont la plupart ont moins de quinze ans.

Un contrat sogdien de Turfan daté de 639 a été publié par Y. Yoshida ${ }^{27}$ :

L'an 16 de Yanchyu, seigneur de Turfan [Chinānchkanth, la «Ville des Chinois»], grand iltebir, le $5^{\mathrm{e}}$ mois chinois qui en sogdien est appelé mois Khshumsafich, l'année du Porc, le $27^{\mathrm{e}}$ jour.

24. « Des Sogdiens et des $\mathrm{Hu} », o p$. cit.

25. É. TROMBERT, « La vigne et le vin en Chine : misères et succès d'une tradition allogène », Journal asiatique, vol. 289, $\mathrm{n}^{\circ}$ 2, 2001, p. 285-327 ; et vol. 290, $\mathrm{n}^{\circ}$ 2, 2002, p. 485-563.

26. CHENG Xu, «The tomb murals of the Tang dynasty recently discovered in the vicinity of the Tang Chang' an city », Wenwu, vol. 12, 2014, p. 64-80 (en chinois).

27. Traduction anglaise chez V. HANSEN, op. cit., p. 181-182, que je reprends d'après l'original. 
Au marché de Turfan, en présence du peuple, le moine Yansyan (Yanxiang) fils d'Uta, de la famille Chan (Zhang), a acheté à Ukhushuvirt fils de Tōdhak, de Samarkand, une fille esclave de la famille Chuyak, née au Turkestan, nommée Opacha, pour 120 drachmes très pures de frappe perse.

Le moine Yansyan a acheté la fille esclave Opacha comme une esclave complètement achetée, sans dettes, sans avoirs, sans poursuites, sans contestation, pour toujours, en propriété pour ses fils, ses petits-fils, sa famille, sa descendance. Qu'en conséquence tant le moine Yansyan lui-même que ses fils, ses petits-fils, sa famille, sa descendance, à leur volonté la battent, la violent [rnp- « attaquer, violer »], l'attachent, la vendent, la mettent en gage, la donnent, le redonnent, et lui fassent tout ce qu'il leur plaira de faire. Au même titre qu'une fille esclave héritée du père, ou du grand-père, ou née dans le clan, ou née à côté, ou née à la maison, cette [esclave] achetée moyennant espèces est propriété permanente.

Et que vis-à -vis de cette fille esclave nommée Opacha, Ukhushuvirt soit dégagé de toute ancienne obligation ou droit de coercition.

[...]

(Liste des témoins, tous sogdiens, issus de quatre royaumes différents).

Ce contrat de fille esclave a été écrit par Ukhwān, fils de Patōr, sous l'autorité de Patōr, scribe en chef, par ordre d'Ukhushuvirt, et avec le consentement d'Opacha.

Le contrat date de l'année précédant la conquête de Turfan par les Tang (iltebir est le titre donné par les Turcs aux souverains locaux subordonnés). L'acheteur est chinois. Comment un moine bouddhique peut-il avoir légalement une concubine et des descendants? Il y avait des ordinations fictives pour échapper au fisc, mais elles se répandront surtout plus tard; par ailleurs les documents de Niya mentionnaient des moines qui vivaient en famille et ne mettaient leur robe que pour les cérémonies. Le nom de la fille, Opacha, ne s'explique pas en sogdien, mais il pourrait s'expliquer en turc : opa «sœur aînée, femme plus âgée », d'où en ouzbek moderne opacha « tantine », terme affectueux pour une jeune fille. Le nom de sa famille ne s'explique pas. Le seul élément clair est qu'elle vient du «Turkestan », donc de territoires tenus directement par les Turcs. Les conditions sous lesquelles elle entre au service, avec son consentement, paraissent terribles, mais le contrat sogdien a au moins la franchise d'étaler ouvertement le fait que, dans tous les systèmes esclavagistes de l'histoire (et jusqu'aux survivances les plus modernes), les esclaves féminines et aussi les garçons, même très jeunes, étaient à la disposition sexuelle des maîtres. Ceci dit, il faut tenir compte aussi des routines juridiques : le propre d'un contrat est de prévoir toutes les situations possibles.

\section{La fin}

Une nouvelle vague d'immigration est intervenue lors de la conquête arabe de la Sogdiane dans la première moitié du VIII ${ }^{\mathrm{e}}$ siècle. En 755-763, la révolte du général turco-sogdien An Lushan ( « Roshan de Boukhara ») s'est appuyée sur ces réseaux, que va faire tomber le brutal ressaisissement chinois qui s'est ensuivi. Les Sogdiens sont victimes de pogroms et, pour survivre, adoptent une stratégie de dissimulation qui les fait disparaître des sources. À l'ouest, ils se fondent dans le royaume turc ouïghour auquel ils apportent une contribution essentielle dans tous les domaines. En Chine, les marchands d'Asie centrale sont toujours présents, mais moins nombreux et désormais aussi dans les ports du Sud ; ils sont maintenant musulmans 

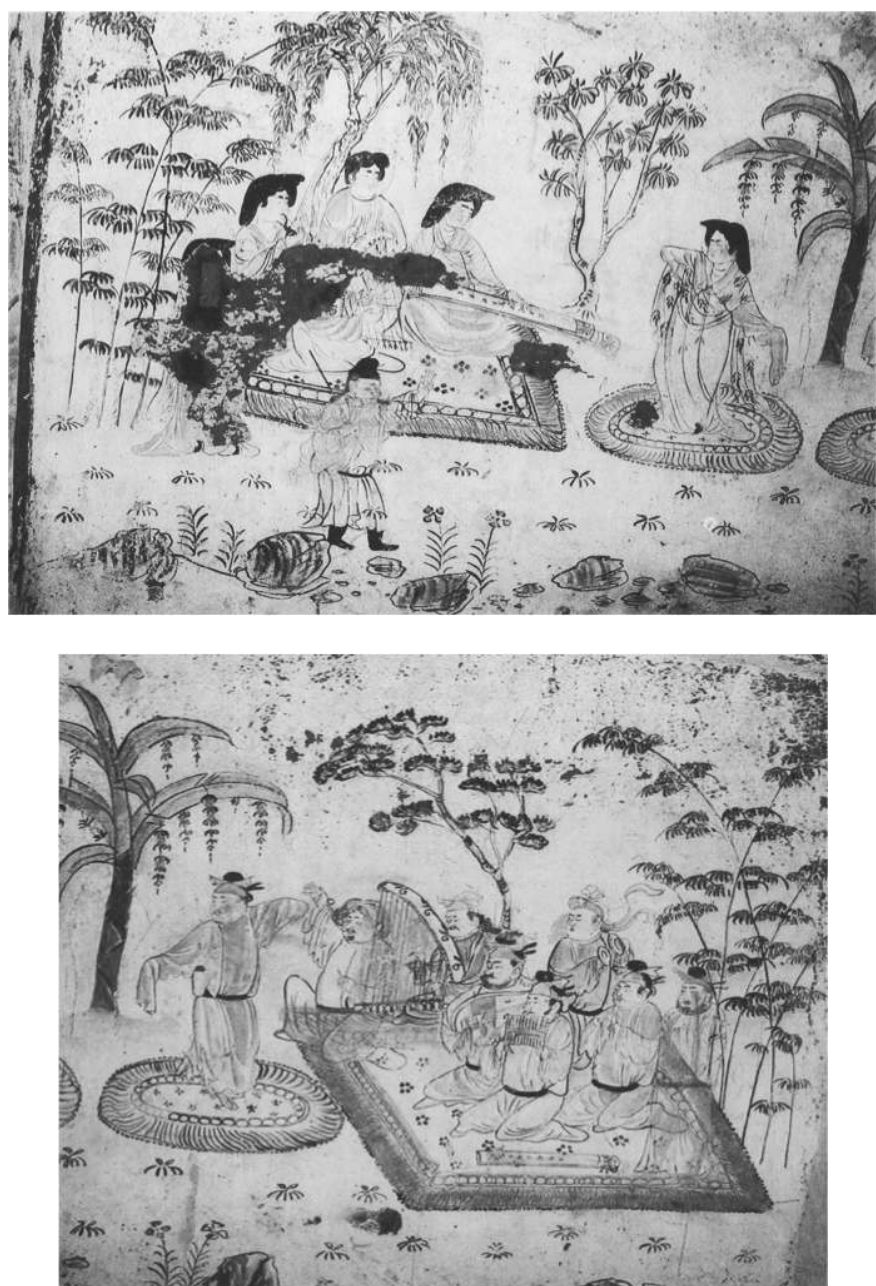

Figures 4a et $\mathbf{4 b}$ - Orchestre chinois et orchestre sogdien. Tombe de Han Xiu, Xi' an, 740. Source : Cheng Xu, Wenwu, 2014 : 12, figures 10-11.

ou juifs ${ }^{28}$, en attendant les Arméniens à partir du $\mathrm{XI}^{\mathrm{e}}$ siècle. Le stéréotype du riche marchand sogdien subsistera longtemps dans la littérature de fiction, et plus longtemps encore dans les formes mineures de l'art ${ }^{29}$.

28. Au IX ${ }^{\mathrm{e}}$ siècle, les documents judéo-persans du Tarim indiquent un substrat sogdien (P. Lurje, conférence au Collège de France, 24 mars 2015).

29. Une statuette de porcelaine polychrome de l'époque Kanxi (1661-1722) exposée au musée Guimet figure encore le cavalier barbare comme un Sogdien de l'époque Tang, de manière 
JouRnÉE D'Études - L'UstrushanA DANS L'ANTIQUITÉ ET LE HAUT MOYEN ÂGE. DES MARGES DE L'CEKOUMĖNE AU CCEUR POLITIQUE DU CALIFAT

Collège de France, 18 mai 2018. Journée d'études co-organisée avec Yury Karev et Claude Rapin (AOROC, ENS/CNRS).

- Claude Rapin, « Note sur l'Ustrushana et ses voisins dans l'Antiquité : Cyreschata, Alexandrie Eschatè, Antioche de Scythie »;

- Sören Stark (ISAW, New York), « Nomadic cultures in Ustrushana, $2^{\text {nd }} c$. BCE$2^{\text {nd }}$ c. CE, and their relationship with the Middle Syr-darya archaeological complex »;

- Muhtar Pardaev (Samarkand, Institut d'archéologie), «La culture urbaine de l'Ustrushana du nord-ouest dans l'Antiquité et le Moyen Âge »;

- Pavel Lurje (musée de l'Ermitage), «The wooden lunette in the Shahristan palace $\left(8^{\text {th }} \mathrm{c}.\right) \gg$;

- Etsuko Kageyama (Nara National Research Institute for Cultural Properties),

« The paintings of the Shahristan palace compared with those of Pendzhikent »;

- Elena Baranova (musée de l'Ermitage), «Restoration of the murals in the corridor of the Shahristan palace »;

- Nikita Semenov (musée de l'Ermitage), «The headgear on the Shahristan paintings »;

- Frantz Grenet et Michael Shenkar (Jérusalem, Hebrew University), « Towards a new interpretation of the paintings from the Small Hall at Shahristan: 1) Compositional and iconographical observations; 2) Tentative identifications in the Kayanid cycle »;

- Aleksej Gritsina (Samarkand, Institut d'archéologie), «Rituels et cultes de l'Ustrushana, d'après l'exemple de Zaamin au Moyen Âge »;

- Yury Karev, «Le pouvoir musulman et les souverains de l'Ustrushana »;

- Antonio Panaino (université de Bologne-Ravenne), «La signification historique de la zone du Yaghnob à la période médiévale ».

\section{COURS À L'EXTÉRIEUR}

Téhéran, Institut des sciences humaines, 7 octobre 2017 : « The Iranian religion in the eastern regions: New archaeological and iconographical discoveries » ;

Shiraz, Université, faculté d'Art, 17 octobre 2017 : « The rediscovery of the court culture of the Qarakhanids (Samarkand, 12th $\mathrm{c}$.) ».

\section{CONFÉRENCIERS INVITÉS}

- Dr. Ching Chao-jung (Ryukoku University, Kyoto) : « Kucha in the $4^{\text {th }}-6^{\text {th }}$ century CE: A kingdom rising on the Silk Road» (13 mars 2018);

- Pr. Nobuyoshi Yamabe (Waseda University) : «An examination through digital restoration of a Sukhāvatī painting of Dunhuang stored at Musée Guimet » (3 mai 2018, à l'invitation conjointe de Jean-Noël Robert).

totalement anachronique. 


\section{RECHERCHE}

Mon travail de recherche continue de s'effectuer pour l'essentiel dans le cadre de l'UMR 8546 Archéologie et philologie d'Orient et d'Occident (AOROC ; CNRS/ ENS, composante de PSL).

La mission archéologique franco-ouzbèke de Sogdiane maintenant dirigée par Claude Rapin se concentre sur la préparation des publications. La mise en ordre des archives de la fouille, notamment la mise en série des rapports annuels (1989-2013), tâche préalable indispensable, s'est effectuée grâce à Svetlana Gorshenina, maître de conférences associée à la chaire jusqu'en août 2018. Trois volumes sont en préparation avancée: B. Lyonnet sur les céramiques, publication financée par l'Académie des inscriptions; Yury Karev sur le palais abbasside; F. Grenet et C. Rapin (dir.) sur les fouilles du site d'Afrasiab.

Toujours en liaison avec le terrain en Ouzbékistan, je participe au comité scientifique de l'expédition archéologique karakalpako-australienne qui fouille au Khorezm (au sud de la mer d'Aral). Je poursuis ma contribution à l'étude et à la publication des peintures murales trouvées par cette mission.

J'ai aussi été amené cette année à travailler sur les phénomènes de transferts artistique entre, d'une part, l'empire romain et l'Égypte romano-byzantine, d'autre part, l'Iran et la Bactriane à l'époque sassanide, dans le cadre de la publication de plats d'argent à décors mythologiques qui se situent à la convergence de ces aires culturelles (A. DAN, F. GRENET et N. Sims-WilliAMS, « Homeric scenes in Bactria and India: Two silver plates with Bactrian and Middle Persian inscriptions », Bulletin of the Asia Institute, vol. 28, 2014 (version numérique : 2018), p. 195-296 ; S. AZARNOUCHE, E. FRAGAKI et F. GRENET, « De l'Égypte à la Perse : un plat sassanide du VII siècle », Études alexandrines. Alexandrina, vol. 5, à paraître en 2020).

\section{PUBLICATIONS}

GRENET F., « More Zoroastrian scenes on the Wirkak (Shi Jun) sarcophagus », Bulletin of the Asia Institute, vol. 27, édition numérique 2017 [2013], p. 1-12.

GRENET F., «The circulation of astrological lore and its political use. Between Roman East, Sasanian Iran, Central Asia, India and the Turks », in N. Di Cosmo et M. MAAS (dir.), Eurasian Empires in Late Antiquity: Rome, China, Iran, and the Steppe, Cambridge/New York, Cambridge University Press, 2018, p. 235-252.

GRENET F., «OPTO $\Sigma$ ПANA $=$ *OPTO IחANA: An etymological attempt $»$, in C. RAPIN et F. GRENET, « How Alexander entered India », Afghanistan, vol. 1, n 1, 2018, p. 173-174.

GRENET F., « Boris Il'ich Marshak i vostochnaja torevtika », préface à B.I. MARSHAK, Istorija vostochnoj torevtiki III-XIII vv. i problemy kul'turnoj preemstvennosti, Saint-Pétersbourg, Akademija issledovanija kul'tury, 2017, p. 14-19. 
